\title{
Studying the Partial Differential Problem Using Maple
}

\author{
Chii-Huei Yu ${ }^{1, *}$, Bing-Huei Chen ${ }^{2}$ \\ ${ }^{1}$ Department of Management and Information, Nan Jeon University of Science and Technology, Tainan City, 73746, Taiwan \\ ${ }^{2}$ Department of Electrical Engineering, Nan Jeon University of Science and Technology, Tainan City, 73746, Taiwan \\ *Corresponding Author: chiihuei@mail.nju.edu.tw
}

Copyright (C 2014 Horizon Research Publishing All rights reserved.

\begin{abstract}
This paper uses the mathematical software Maple for the auxiliary tool to study the partial differential problem of four types of multivariable functions. We can obtain the infinite series forms of any order partial derivatives of these four types of multivariable functions by using differentiation term by term theorem, and hence greatly reduce the difficulty of calculating their higher order partial derivative values. On the other hand, we propose some examples to do calculation practically. The research methods adopted in this study involved finding solutions through manual calculations and verifying these solutions by using Maple. This type of research method not only allows the discovery of calculation errors, but also helps modify the original directions of thinking from manual and Maple calculations. For this reason, Maple provides insights and guidance regarding problem-solving methods.
\end{abstract}

Keywords Partial Derivatives, Infinite Series Forms, Differentiation Term By Term Theorem, Maple

\section{Introduction}

As information technology advances, whether computers can become comparable with human brains to perform abstract tasks, such as abstract art similar to the paintings of Picasso and musical compositions similar to those of Beethoven, is a natural question. Currently, this appears unattainable. In addition, whether computers can solve abstract and difficult mathematical problems and develop abstract mathematical theories such as those of mathematicians also appears unfeasible. Nevertheless, in seeking for alternatives, we can study what assistance mathematical software can provide. This study introduces how to conduct mathematical research using the mathematical software Maple. The main reasons of using Maple in this study are its simple instructions and ease of use, which enable beginners to learn the operating techniques in a short period. By employing the powerful computing capabilities of Maple, difficult problems can be easily solved. Even when Maple cannot determine the solution, problem-solving hints can be identified and inferred from the approximate values calculated and solutions to similar problems, as determined by Maple. For this reason, Maple can provide insights into scientific research. Inquiring through an online support system provided by Maple or browsing the Maple website (www.maplesoft.com) can facilitate further understanding of Maple and might provide unexpected insights. For the instructions and operations of Maple, [1-7] can be adopted as references.

In calculus and engineering mathematics curricula, evaluating the $m$-th order partial derivative value of a multivariable function at some point, in general, needs to go through two procedures: firstly determining the $m$-th order partial derivative of this function, and then taking the point into this $m$-th order partial derivative. These two procedures will make us face with increasingly complex calculations when calculating higher order partial derivative values ( i.e. $m$ is large), and hence to obtain the answers by manual calculations is not easy. In this paper, we study the partial differential problem of the following four types of $n$-variables functions

$$
\begin{array}{r}
f\left(x_{1}, x_{2}, \cdots, x_{n}\right)=\prod_{k=1}^{n} x_{k}{ }^{r_{k}} \cdot \sin ^{-1}\left(\prod_{k=1}^{n} x_{k} s_{k}\right) \\
g\left(x_{1}, x_{2}, \cdots, x_{n}\right)=\prod_{k=1}^{n} x_{k}{ }^{r_{k}} \cdot \cos ^{-1}\left(\prod_{k=1}^{n} x_{k}{ }^{s_{k}}\right) \\
p\left(x_{1}, x_{2}, \cdots, x_{n}\right)=\prod_{k=1}^{n} x_{k}^{r_{k}} \cdot \csc ^{-1}\left(\prod_{k=1}^{n} x_{k}{ }^{s} k\right) \\
q\left(x_{1}, x_{2}, \cdots, x_{n}\right)=\prod_{k=1}^{n} x_{k}{ }^{r_{k}} \cdot \sec ^{-1}\left(\prod_{k=1}^{n} x_{k} s_{k}\right)
\end{array}
$$

Where $n$ is a positive integer, $r_{k}, s_{k}$ are real numbers for all $k=1, \ldots, n$. We can obtain the infinite series forms of any order partial derivatives of these four types of $n$ -variables functions by using differentiation term by term theorem ; these are the major results of this study (i.e., Theorems 1-4), and hence greatly reduce the difficulty of calculating their higher order partial derivative values. For the study of related partial differential problems can refer to [8-19]. In addition, we provide some examples to do 
calculation practically. The research methods adopted in this study involved finding solutions through manual calculations and verifying these solutions by using Maple. This type of research method not only allows the discovery of calculation errors, but also helps modify the original directions of thinking from manual and Maple calculations. Therefore, Maple provides insights and guidance regarding problem-solving methods.

\section{Main Results}

Firstly, we introduce some notations and formulas used in this paper.

\subsection{Notations}

2.1.1. $\prod_{k=1}^{n} a_{k}=a_{1} \times a_{2} \times \cdots \times a_{n}$, where $n$ is a positive integer, $a_{k}$ are real numbers for all $k=1, . ., n$.

2.1.2. Suppose $r$ is any real number, $m$ is any positive integer. Define $(r)_{m}=r(r-1) \cdots(r-m+1)$, and $(r)_{0}=1$.

2.1.3. Suppose $m, n$ are positive integers, we define

$$
\left(\begin{array}{c}
m \\
n
\end{array}\right)=\frac{m !}{n !(m-n) !} \text {, and }\left(\begin{array}{c}
m \\
0
\end{array}\right)=1 .
$$

2.1.4. Suppose $n$ is a positive integer, $j_{k}$ are non-negative integers for all $k=1, . ., n$. For the $n$-variables function $f\left(x_{1}, x_{2}, \cdots, x_{n}\right)$, its $j_{k}$-times partial derivative with respect to $x_{k}$ for all $k=1, \ldots, n$, forms a $j_{1}+j_{2}+\cdots+j_{n}$-th order partial derivative, and denoted by $\frac{\partial^{j_{1}+j_{2}+\cdots+j_{n}} f}{\partial x_{n}^{j_{n}} \cdots \partial x_{2}^{j_{2}} \partial x_{1}^{j_{1}}}\left(x_{1}, x_{2}, \cdots, x_{n}\right)$.

\subsection{Formulas ([20])}

2.2.1. The inverse sine function

$$
\sin ^{-1} y=\sum_{i=0}^{\infty} \frac{\left(\begin{array}{c}
2 i \\
i
\end{array}\right)}{4^{i}(2 i+1)} y^{2 i+1} \text {, where } y \text { is a real number, }|y|<1 .
$$

2.2.2. The inverse cosine function

$$
\cos ^{-1} y=\frac{\pi}{2}-\sum_{i=0}^{\infty} \frac{\left(\begin{array}{c}
2 i \\
i
\end{array}\right)}{4^{i}(2 i+1)} y^{2 i+1} \text {, where } y \text { is a real number, }|y|<1 .
$$

2.2.3. The inverse cosecant function

$$
\csc ^{-1} y=\sum_{i=0}^{\infty} \frac{\left(\begin{array}{c}
2 i \\
i
\end{array}\right)}{4^{i}(2 i+1)} y^{-(2 i+1)} \text {, where } y \text { is a real number, }|y|>1 .
$$

2.2.4. The inverse secant function

$$
\sec ^{-1} y=\frac{\pi}{2}-\sum_{i=0}^{\infty} \frac{\left(\begin{array}{c}
2 i \\
i
\end{array}\right)}{4^{i}(2 i+1)} y^{-(2 i+1)} \text {, where } y \text { is a real number, }|y|>1 .
$$

Next, we introduce an important theorem used in this study.

\subsection{Differentiation Term by Term Theorem ([21])}


If, for all non-negative integer $k$, the functions $g_{k}:(a, b) \rightarrow R$ satisfy the following three conditions: (i) there exists a point $x_{0} \in(a, b)$ such that $\sum_{k=0}^{\infty} g_{k}\left(x_{0}\right)$ is convergent, (ii) all functions $g_{k}(x)$ are differentiable on open interval $(a, b)$, (iii) $\sum_{k=0}^{\infty} \frac{d}{d x} g_{k}(x)$ is uniformly convergent on $(a, b)$. Then $\sum_{k=0}^{\infty} g_{k}(x)$ is uniformly convergent and differentiable on $(a, b)$. Moreover, its derivative $\frac{d}{d x} \sum_{k=0}^{\infty} g_{k}(x)=\sum_{k=0}^{\infty} \frac{d}{d x} g_{k}(x)$.

The following is the first result in this study, we determine the infinite series forms of any order partial derivatives of the multivariable function (1).

2.4. Theorem 1 Suppose $n$ is a positive integer, $r_{k}, s_{k}$ are real numbers, and $j_{k}$ are non-negative integers for all $k=1, . ., n$. If the $n$-variables function

$$
f\left(x_{1}, x_{2}, \cdots, x_{n}\right)=\prod_{k=1}^{n} x_{k}{ }^{r} \cdot \sin ^{-1}\left(\prod_{k=1}^{n} x_{k} s_{k}\right)
$$

satisfies $x_{k}{ }^{r}{ }^{k}, x_{k}{ }^{s} k$ exist, $x_{k} \neq 0$ for all $k=1, . ., n$, and $\left|\prod_{k=1}^{n} x_{k}{ }^{s k}\right|<1$. Then the $j_{1}+j_{2}+\cdots+j_{n}$-th order partial derivative of $f\left(x_{1}, x_{2}, \cdots, x_{n}\right)$,

$$
\begin{gathered}
\frac{\partial^{j_{1}+j_{2}+\cdots+j_{n}} f}{\partial x_{n}{ }^{j_{n}} \cdots \partial x_{2}{ }^{j_{2}} \partial x_{1}{ }^{j_{1}}}\left(x_{1}, x_{2}, \cdots, x_{n}\right) \\
=\sum_{i=0}^{\infty} \frac{\left(\begin{array}{c}
2 i \\
4^{i}
\end{array}\right)}{4^{i}(2 i+1)} \prod_{k=1}^{n}\left((2 i+1) s_{k}+r_{k}\right)_{j k} \prod_{k=1}^{n} x_{k}^{(2 i+1) s_{k}+r_{k}-j_{k}}
\end{gathered}
$$

2.4.1. Proof

$$
\begin{aligned}
\text { Because } & f\left(x_{1}, x_{2}, \cdots, x_{n}\right) \\
= & \prod_{k=1}^{n} x_{k}{ }^{r_{k}} \cdot \sin ^{-1}\left(\prod_{k=1}^{n} x_{k}{ }^{s} k\right) \\
= & \prod_{k=1}^{n} x_{k}{ }^{r k} \cdot \sum_{i=0}^{\infty} \frac{\left(\begin{array}{c}
2 i \\
i
\end{array}\right)}{4^{i}(2 i+1)}\left(\prod_{k=1}^{n} x_{k} s_{k}\right)^{2 i+1} \\
= & \sum_{i=0}^{\infty} \frac{\left(\begin{array}{c}
2 i \\
i
\end{array}\right)}{4^{i}(2 i+1)} \prod_{k=1}^{n} x_{k}^{(2 i+1) s_{k}+r_{k}}
\end{aligned}
$$

By differentiation term by term theorem, differentiating $j_{k}$-times with respect to $x_{k}(k=1, . ., n)$ on both sides of (6), we obtain the $j_{1}+j_{2}+\cdots+j_{n}$-th order partial derivative of $f\left(x_{1}, x_{2}, \cdots, x_{n}\right)$,

$$
\frac{\partial^{j_{1}+j_{2}+\cdots+j_{n}} f}{\partial x_{n}^{j_{n} \cdots \partial x_{2}{ }^{j_{2}} \partial x_{1}^{j_{1}}}}\left(x_{1}, x_{2}, \cdots, x_{n}\right)
$$




$$
=\sum_{i=0}^{\infty} \frac{\left(\begin{array}{c}
2 i \\
i
\end{array}\right)}{4^{i}(2 i+1)} \prod_{k=1}^{n}\left((2 i+1) s_{k}+r_{k}\right)_{j k} \prod_{k=1}^{n} x_{k}^{(2 i+1) s_{k}+r_{k}-j_{k}}
$$

Next, we determine the infinite series forms of any order partial derivatives of the multivariable function (2).

2.5. Theorem 2 Let the assumptions be the same as Theorem 1. Suppose the $n$-variables function

$$
g\left(x_{1}, x_{2}, \cdots, x_{n}\right)=\prod_{k=1}^{n} x_{k}^{r_{k}} \cdot \cos ^{-1}\left(\prod_{k=1}^{n} x_{k} s_{k}\right)
$$

satisfies $x_{k}{ }^{r}{ }_{k}, x_{k}{ }^{s_{k}}$ exist, $x_{k} \neq 0$ for all $k=1, . ., n$, and $\left|\prod_{k=1}^{n} x_{k}{ }^{s}\right|<1$. Then the $j_{1}+j_{2}+\cdots+j_{n}$-th order partial derivative of $g\left(x_{1}, x_{2}, \cdots, x_{n}\right)$,

$$
\begin{gathered}
\frac{\partial^{j_{1}+j_{2}+\cdots+j_{n}} g}{\partial x_{n}{ }^{j_{n}} \cdots \partial x_{2}{ }^{j} \partial x_{1} j_{1}}\left(x_{1}, x_{2}, \cdots, x_{n}\right) \\
=\frac{\pi}{2} \cdot \prod_{k=1}^{n}\left(r_{k}\right)_{j k} \cdot \prod_{k=1}^{n} x_{k}{ }^{r_{k}-j_{k}}-\sum_{i=0}^{\infty} \frac{\left(\begin{array}{c}
2 i \\
i
\end{array}\right)}{4^{i}(2 i+1)} \prod_{k=1}^{n}\left((2 i+1) s_{k}+r_{k}\right)_{j k} \prod_{k=1}^{n} x_{k}(2 i+1) s_{k}+r_{k}-j_{k}
\end{gathered}
$$

2.5.1. Proof

$$
\text { Because } \begin{aligned}
& g\left(x_{1}, x_{2}, \cdots, x_{n}\right) \\
= & \prod_{k=1}^{n} x_{k}{ }^{r_{k}} \cdot \cos ^{-1}\left(\prod_{k=1}^{n} x_{k}{ }^{s} k\right) \\
= & \prod_{k=1}^{n} x_{k}{ }^{r_{k}} \cdot\left[\frac{\pi}{2}-\sum_{i=0}^{\infty} \frac{\left(\begin{array}{c}
2 i \\
i
\end{array}\right)}{4^{i}(2 i+1)}\left(\prod_{k=1}^{n} x_{k}{ }^{s}\right)^{2 i+1}\right] \\
= & \frac{\pi}{2} \cdot \prod_{k=1}^{n} x_{k}{ }^{r_{k}}-\sum_{i=0}^{\infty} \frac{\left(\begin{array}{c}
2 i \\
i
\end{array}\right)}{4^{i}(2 i+1)} \prod_{k=1}^{n} x_{k}^{(2 i+1) s_{k}+r_{k}}
\end{aligned}
$$

Using differentiation term by term theorem, differentiating $j_{k}$-times with respect to $x_{k} \quad(k=1, . ., n)$ on both sides of $(8)$, we can determine the $j_{1}+j_{2}+\cdots+j_{n}$-th order partial derivative of $g\left(x_{1}, x_{2}, \cdots, x_{n}\right)$,

$$
\begin{gathered}
\frac{\partial^{j_{1}+j_{2}+\cdots+j_{n}} g}{\partial x_{n}^{j_{n}} \cdots \partial x_{2}{ }^{j 2} \partial x_{1} j_{1}}\left(x_{1}, x_{2}, \cdots, x_{n}\right) \\
=\frac{\pi}{2} \cdot \prod_{k=1}^{n}\left(r_{k}\right)_{j_{k}} \cdot \prod_{k=1}^{n} x_{k}{ }^{r_{k}-j_{k}}-\sum_{i=0}^{\infty} \frac{\left(\begin{array}{c}
2 i \\
i
\end{array}\right)}{4^{i}(2 i+1)} \prod_{k=1}^{n}\left((2 i+1) s_{k}+r_{k}\right)_{j k} \prod_{k=1}^{n} x_{k}(2 i+1) s_{k}+r_{k}-j_{k}
\end{gathered}
$$

In the following, we determine the infinite series forms of any order partial derivatives of the multivariable function (3). 
2.6. Theorem 3 If the assumptions are the same as Theorem 1. Suppose the $n$-variables function

$$
p\left(x_{1}, x_{2}, \cdots, x_{n}\right)=\prod_{k=1}^{n} x_{k}^{r_{k}} \cdot \csc ^{-1}\left(\prod_{k=1}^{n} x_{k} s_{k}\right)
$$

satisfies $x_{k}{ }^{r_{k}}, x_{k}{ }^{s_{k}}$ exist, $x_{k} \neq 0$ for all $k=1, . ., n$, and $\left|\prod_{k=1}^{n} x_{k} s_{k}\right|>1$. Then the $j_{1}+j_{2}+\cdots+j_{n}$-th order partial derivative of $p\left(x_{1}, x_{2}, \cdots, x_{n}\right)$,

$$
\begin{gathered}
\frac{\partial^{j_{1}+j_{2}+\cdots+j_{n}} p}{\partial x_{n}{ }^{j_{n}} \cdots \partial x_{2}{ }^{j 2} \partial x_{1} j_{1}}\left(x_{1}, x_{2}, \cdots, x_{n}\right) \\
=\sum_{i=0}^{\infty} \frac{\left(\begin{array}{c}
2 i \\
i
\end{array}\right)}{4^{i}(2 i+1)} \prod_{k=1}^{n}\left(-(2 i+1) s_{k}+r_{k}\right) j_{k} \prod_{k=1}^{n} x_{k}^{-(2 i+1) s_{k}+r_{k}-j_{k}}
\end{gathered}
$$

2.6.1. Proof

$$
\begin{aligned}
& \text { Because } \quad p\left(x_{1}, x_{2}, \cdots, x_{n}\right) \\
& =\prod_{k=1}^{n} x_{k}{ }^{r_{k}} \cdot \csc ^{-1}\left(\prod_{k=1}^{n} x_{k}{ }_{k}\right) \\
& =\prod_{k=1}^{n} x_{k}{ }^{r} k \cdot \sum_{i=0}^{\infty} \frac{\left(\begin{array}{c}
2 i \\
i
\end{array}\right)}{4^{i}(2 i+1)}\left(\prod_{k=1}^{n} x_{k} s_{k}\right)^{-(2 i+1)} \\
& =\sum_{i=0}^{\infty} \frac{\left(\begin{array}{c}
2 i \\
i
\end{array}\right)}{4^{i}(2 i+1)} \cdot \prod_{k=1}^{n} x_{k}^{-(2 i+1) s_{k}+r_{k}}
\end{aligned}
$$

Using differentiation term by term theorem, differentiating $j_{k}$-times with respect to $x_{k} \quad(k=1, . ., n)$ on both sides of $(10)$, we obtain the $j_{1}+j_{2}+\cdots+j_{n}$-th order partial derivative of $p\left(x_{1}, x_{2}, \cdots, x_{n}\right)$,

$$
\begin{aligned}
& \frac{\partial^{j_{1}+j_{2}+\cdots+j_{n}} p}{\partial x_{n}{ }^{j_{n}} \cdots \partial x_{2}^{j 2} \partial x_{1}{ }^{j 1}}\left(x_{1}, x_{2}, \cdots, x_{n}\right) \\
& \quad=\sum_{i=0}^{\infty} \frac{\left(\begin{array}{c}
2 i \\
i
\end{array}\right)}{4^{i}(2 i+1)} \prod_{k=1}^{n}\left(-(2 i+1) s_{k}+r_{k}\right) j_{k} \prod_{k=1}^{n} x_{k}^{-(2 i+1) s_{k}+r_{k}-j_{k}}
\end{aligned}
$$

2.7. Theorem 4 Let the assumptions be the same as Theorem 1. If the $n$-variables function

$$
q\left(x_{1}, x_{2}, \cdots, x_{n}\right)=\prod_{k=1}^{n} x_{k}{ }^{r} \cdot \sec ^{-1}\left(\prod_{k=1}^{n} x_{k} s_{k}\right)
$$

satisfies $x_{k}{ }^{r}, x_{k}{ }^{s} k$ exist, $x_{k} \neq 0$ for all $k=1, . ., n$, and $\left|\prod_{k=1}^{n} x_{k} s_{k}\right|>1$. Then the $j_{1}+j_{2}+\cdots+j_{n}$-th order partial derivative of $q\left(x_{1}, x_{2}, \cdots, x_{n}\right)$,

$$
\frac{\partial^{j_{1}+j_{2}+\cdots+j_{n}} q}{\partial x_{n}^{j_{n}} \cdots \partial x_{2}^{j 2} \partial x_{1}^{j 1}}\left(x_{1}, x_{2}, \cdots, x_{n}\right)
$$




$$
=\frac{\pi}{2} \cdot \prod_{k=1}^{n}\left(r_{k}\right)_{j_{k}} \cdot \prod_{k=1}^{n} x_{k}{ }^{r_{k}-j_{k}}-\sum_{i=0}^{\infty} \frac{\left(\begin{array}{c}
2 i \\
4^{i}
\end{array}\right)}{4^{i}(2 i+1)} \prod_{k=1}^{n}\left(-(2 i+1) s_{k}+r_{k}\right) j_{k} \prod_{k=1}^{n} x_{k}-(2 i+1) s_{k}+r_{k}-j_{k}
$$

2.7.1. Proof

$$
\text { Because } \begin{aligned}
& q\left(x_{1}, x_{2}, \cdots, x_{n}\right) \\
= & \prod_{k=1}^{n} x_{k}{ }^{r_{k}} \cdot \sec ^{-1}\left(\prod_{k=1}^{n} x_{k}{ }^{s}\right) \\
= & \prod_{k=1}^{n} x_{k}{ }^{r_{k}} \cdot\left[\frac{\pi}{2}-\sum_{i=0}^{\infty} \frac{\left(\begin{array}{c}
2 i \\
i
\end{array}\right)}{4^{i}(2 i+1)}\left(\prod_{k=1}^{n} x_{k}{ }^{s}\right)^{-(2 i+1)}\right] \\
= & \frac{\pi}{2} \cdot \prod_{k=1}^{n} x_{k}{ }^{r_{k}}-\sum_{i=0}^{\infty} \frac{\left(\begin{array}{c}
2 i \\
i
\end{array}\right)}{4^{i}(2 i+1)} \cdot \prod_{k=1}^{n} x_{k}^{-(2 i+1) s_{k}+r_{k}}
\end{aligned}
$$

By differentiation term by term theorem, differentiating $j_{k}$-times with respect to $x_{k}(k=1, . ., n)$ on both sides of (12), we obtain the $j_{1}+j_{2}+\cdots+j_{n}$-th order partial derivative of $q\left(x_{1}, x_{2}, \cdots, x_{n}\right)$,

$$
\begin{gathered}
\frac{\partial^{j_{1}+j_{2}+\cdots+j_{n}} q}{\partial x_{n}^{j_{n}} \cdots \partial x_{2}{ }^{j 2} \partial x_{1}{ }^{j_{1}}}\left(x_{1}, x_{2}, \cdots, x_{n}\right) \\
=\frac{\pi}{2} \cdot \prod_{k=1}^{n}\left(r_{k}\right)_{j_{k}} \cdot \prod_{k=1}^{n} x_{k}^{r_{k}-j_{k}}-\sum_{i=0}^{\infty} \frac{\left(\begin{array}{c}
2 i \\
i
\end{array}\right)}{4^{i}(2 i+1)} \prod_{k=1}^{n}\left(-(2 i+1) s_{k}+r_{k}\right)_{j k} \prod_{k=1}^{n} x_{k}-(2 i+1) s_{k}+r_{k}-j_{k}
\end{gathered}
$$

\section{Examples}

Next, for the partial differential problem of the four types of multivariable functions in this study, we provide four examples and use Theorems 1-4 to determine the infinite series forms of any order partial derivatives and some higher order partial derivative values of these functions. On the other hand, we employ Maple to calculate the approximations of these higher order partial derivative values and their solutions for verifying our answers.

3.1. Example 1 Assume the domain of the two-variables function

$$
f\left(x_{1}, x_{2}\right)=x_{1}{ }^{-3 / 8} x_{2}{ }^{11 / 7} \sin ^{-1}\left(x_{1}{ }^{9 / 4} x_{2}{ }^{-4 / 5}\right)
$$

is $\left\{\left(x_{1}, x_{2}\right) \in R^{2}\left|x_{1}>0, x_{2} \neq 0,\right| x_{1}{ }^{9 / 4} x_{2}{ }^{-4 / 5} \mid<1\right\}$. By Theorem 1, we obtain any $j_{1}+j_{2}$-th order partial derivative of $f\left(x_{1}, x_{2}\right)$,

$$
\begin{gathered}
\frac{\partial^{j_{1}+j_{2}} f}{\partial x_{2}{ }^{j_{2}} \partial x_{1}{ }^{j_{1}}}\left(x_{1}, x_{2}\right) \\
=\sum_{i=0}^{\infty} \frac{\left(\begin{array}{c}
2 i \\
i
\end{array}\right)}{4^{i}(2 i+1)}\left(\frac{9}{2} i+\frac{15}{8}\right)_{j 1}\left(-\frac{8}{5} i+\frac{27}{35}\right)_{j 2} \cdot x_{1} \frac{9}{2} i+\frac{15}{8}-j_{1} \cdot x_{2}-\frac{8}{5} i+\frac{27}{35}-j_{2}
\end{gathered}
$$


For all $x_{1}>0, x_{2} \neq 0,\left|x_{1}{ }^{9 / 4} x_{2}{ }^{-4 / 5}\right|<1$. Hence, we can evaluate the 10-th order partial derivative value of $f\left(x_{1}, x_{2}\right)$ at $\left(\frac{2}{3}, \frac{7}{4}\right)$,

$$
\begin{gathered}
\frac{\partial^{10} f}{\partial x_{2}^{3} \partial x_{1}{ }^{7}}\left(\frac{2}{3}, \frac{7}{4}\right) \\
=\sum_{i=0}^{\infty} \frac{\left(\begin{array}{c}
2 i \\
i
\end{array}\right)}{4^{i}(2 i+1)}\left(\frac{9}{2} i+\frac{15}{8}\right)_{7}\left(-\frac{8}{5} i+\frac{27}{35}\right)_{3} \cdot\left(\frac{2}{3}\right)^{\frac{9}{2} i-\frac{41}{8}} \cdot\left(\frac{7}{4}\right)^{-\frac{8}{5} i-\frac{78}{35}}
\end{gathered}
$$

We use Maple to verify the correctness of (15) as follows:

$>\mathrm{f}:=(\mathrm{x} 1, \mathrm{x} 2)->\mathrm{x} 1^{\wedge}(-3 / 8)^{*} \mathrm{x} 2^{\wedge}(11 / 7)^{*} \arcsin \left(\mathrm{x} 1^{\wedge}(9 / 4) * \mathrm{x} 2^{\wedge}(-4 / 5)\right)$;

$$
f:=(x 1, x 2) \rightarrow \frac{x 2^{11 / 7} \arcsin \left(\frac{x 1^{9 / 4}}{x 2^{4 / 5}}\right)}{x 1^{3 / 8}}
$$

>evalf(D[1\$7,2\$3](f)(2/3,7/4),14);

$$
-4.2411980514662 \cdot 10^{5}
$$

$>\operatorname{evalf}\left(\operatorname{sum}\left(1 /\left(4 \wedge \mathrm{i}^{*}(2 * \mathrm{i}+1)\right) *(2 * \mathrm{i}) ! /(\mathrm{i} ! * \mathrm{i} !) * \operatorname{product}(9 * \mathrm{i} / 2+15 / 8-\mathrm{j}, \mathrm{j}=0 . .6) * \operatorname{product}(-8 * \mathrm{i} / 5+27 / 35-\mathrm{p}, \mathrm{p}=0 . .2)^{*}(2 / 3)^{\wedge}(9 * \mathrm{i} / 2-41 / 8)\right.\right.$ $*(7 / 4)^{\wedge}(-8 * \mathrm{i} / 5-78 / 35), \mathrm{i}=0$..infinity), 14$)$;

$$
-4.2411980514663 \cdot 10^{5}
$$

3.2. Example 2 Suppose the domain of the three-variables function

$$
g\left(x_{1}, x_{2}, x_{3}\right)=x_{1}{ }^{12 / 5} x_{2}{ }^{-1 / 9} x_{3}{ }^{-7 / 6} \cos ^{-1}\left(x_{1}{ }^{-8 / 3} x_{2}{ }^{10 / 7} x_{3}{ }^{3 / 4}\right)
$$

is $\left\{\left(x_{1}, x_{2}, x_{3}\right) \in R^{3}\left|x_{1} \neq 0, x_{2} \neq 0, x_{3}>0,\right| x_{1}{ }^{-8 / 3} x_{2}{ }^{10 / 7} x_{3}{ }^{3 / 4} \mid<1\right\}$. Using Theorem 2, we have any $j_{1}+j_{2}+j_{3}$-th order partial derivative of $g\left(x_{1}, x_{2}, x_{3}\right)$,

$$
\begin{gathered}
\frac{\partial^{j_{1}+j_{2}+j_{3}} g}{\partial x_{3}{ }^{j_{3}} \partial x_{2}{ }^{j_{2}} \partial x_{1}{ }^{j_{1}}}\left(x_{1}, x_{2}, x_{3}\right) \\
=\frac{\pi}{2} \cdot\left(\frac{12}{5}\right)_{j_{1}}\left(\frac{-1}{9}\right)_{j_{2}}\left(\frac{-7}{6}\right)_{j_{3}} x_{1}^{\frac{12}{5}-j_{1}} \cdot x_{2} \frac{-1}{9}-j_{2} \cdot x_{3} \frac{-7}{6}-j_{3} \\
-\sum_{i=0}^{\infty} \frac{\left(\begin{array}{c}
2 i \\
i
\end{array}\right)}{4^{i}(2 i+1)}\left(-\frac{16}{3} i-\frac{4}{15}\right)_{j_{1}}\left(\frac{20}{7} i+\frac{83}{63}\right)_{j_{2}}\left(\frac{3}{2} i-\frac{5}{12}\right)_{j_{3}} \cdot x_{1}{ }^{-\frac{16}{3} i-\frac{4}{15}-j_{1}} \cdot x_{2} \frac{20}{7} i+\frac{83}{63}-j_{2} \cdot x_{3} \frac{3}{2} i-\frac{5}{12}-j_{3}
\end{gathered}
$$

For all $x_{1} \neq 0, x_{2} \neq 0, x_{3}>0,\left|x_{1}{ }^{-8 / 3} x_{2}{ }^{10 / 7} x_{3}{ }^{3 / 4}\right|<1$. Thus, we can determine the 14 -th order partial derivative value of $g\left(x_{1}, x_{2}, x_{3}\right)$ at $\left(\frac{6}{5}, \frac{4}{9}, \frac{3}{7}\right)$,

$$
\frac{\partial^{14} g}{\partial x_{3}^{6} \partial x_{2}^{5} \partial x_{1}^{3}}\left(\frac{6}{5}, \frac{4}{9}, \frac{3}{7}\right)
$$




$$
\begin{gathered}
=\frac{\pi}{2} \cdot\left(\frac{12}{5}\right)_{3}\left(\frac{-1}{9}\right)_{5}\left(\frac{-7}{6}\right)_{6} \cdot\left(\frac{6}{5}\right)^{-\frac{3}{5}} \cdot\left(\frac{4}{9}\right)^{-\frac{46}{9}} \cdot\left(\frac{3}{7}\right)^{-\frac{43}{6}} \\
-\sum_{i=0}^{\infty} \frac{\left(\begin{array}{c}
2 i \\
i
\end{array}\right)}{4^{i}(2 i+1)}\left(-\frac{16}{3} i-\frac{4}{15}\right)_{3}\left(\frac{20}{7} i+\frac{83}{63}\right)_{5}\left(\frac{3}{2} i-\frac{5}{12}\right)_{6} \cdot\left(\frac{6}{5}\right)^{-\frac{16}{3} i-\frac{49}{15}} \cdot\left(\frac{4}{9}\right)^{\frac{20}{7} i-\frac{232}{63}} \cdot\left(\frac{3}{7}\right)^{\frac{3}{2} i-\frac{77}{12}}
\end{gathered}
$$

Using Maple to verify the correctness of (18) as follows:

$>\mathrm{g}:=(\mathrm{x} 1, \mathrm{x} 2, \mathrm{x} 3)->\mathrm{x} 1^{\wedge}(12 / 5)^{*} \mathrm{x} 2^{\wedge}(-1 / 9) * \mathrm{x} 3^{\wedge}(-7 / 6) * \arccos \left(\mathrm{x} 1^{\wedge}(-8 / 3) * \mathrm{x} 2^{\wedge}(10 / 7) * \mathrm{x} 3^{\wedge}(3 / 4)\right)$;

$$
g:=(x 1, x 2, x 3) \rightarrow \frac{x 1^{12 / 5} \arccos \left(\frac{x 2^{10 / 7} x 3^{3 / 4}}{x 1^{8 / 3}}\right)}{x 2^{1 / 9} x 3^{7 / 6}}
$$

$>\operatorname{evalf}(\mathrm{D}[1 \$ 3,2 \$ 5,3 \$ 6](\mathrm{g})(6 / 5,4 / 9,3 / 7), 14)$

\section{$-1.8324786619206 \cdot 10^{8}$}

$>\operatorname{evalf}\left(\mathrm{Pi} / 2 * \operatorname{product}(12 / 5-\mathrm{r}, \mathrm{r}=0 . .2)^{*} \operatorname{product}(-1 / 9-\mathrm{s}, \mathrm{s}=0 . .4)^{*} \operatorname{product}(-7 / 6-\mathrm{t}, \mathrm{t}=0 . .5)^{*}(6 / 5)^{\wedge}(-3 / 5)^{*}(4 / 9)^{\wedge}(-46 / 9)^{*}(3 / 7)^{\wedge}(-43 / 6)-\right.$ $\operatorname{sum}\left(1 /\left(4 \wedge \mathrm{i}^{*}(2 * \mathrm{i}+1)\right) *(2 * \mathrm{i}) ! /(\mathrm{i} ! * \mathrm{i} !) * \operatorname{product}(-16 * \mathrm{i} / 3-4 / 15-\mathrm{j}, \mathrm{j}=0 . .2) * \operatorname{product}(20 * \mathrm{i} / 7+83 / 63-\mathrm{p}, \mathrm{p}=0 . .4) * \operatorname{product}(3 * \mathrm{i} / 2-5 / 12-\mathrm{q}, \mathrm{q}=\right.$ $0 . .5)^{*}(6 / 5)^{\wedge}(-16 * \mathrm{i} / 3-49 / 15) *(4 / 9)^{\wedge}(20 * \mathrm{i} / 7-232 / 63) *(3 / 7)^{\wedge}(3 * \mathrm{i} / 2-77 / 12), \mathrm{i}=0$. infinity $\left.), 14\right)$;

\section{$-1.8324786619206 \cdot 10^{8}$}

3.3. Example 3 If the domain of the two-variables function

$$
p\left(x_{1}, x_{2}\right)=x_{1}{ }^{9 / 2} x_{2}{ }^{-7 / 8} \csc ^{-1}\left(x_{1}{ }^{-3 / 10} x_{2}{ }^{-6 / 5}\right)
$$

is $\left\{\left(x_{1}, x_{2}\right) \in R^{2}\left|x_{1}>0, x_{2}>0,\right| x_{1}^{-3 / 10} x_{2}{ }^{-6 / 5} \mid>1\right\}$. By Theorem 3, we can evaluate any $j_{1}+j_{2}$-th order partial derivative of $p\left(x_{1}, x_{2}\right)$,

$$
\begin{gathered}
\frac{\partial^{j_{1}+j_{2}} p}{\partial x_{2}{ }^{j_{2}} \partial x_{1} j_{1}}\left(x_{1}, x_{2}\right) \\
=\sum_{i=0}^{\infty} \frac{\left(\begin{array}{c}
2 i \\
i
\end{array}\right)}{4^{i}(2 i+1)}\left(\frac{3}{5} i+\frac{24}{5}\right)_{j_{1}}\left(\frac{12}{5} i+\frac{13}{40}\right)_{j_{2}} \cdot x_{1} \frac{3}{5} i+\frac{24}{5}-j_{1} \cdot x_{2} \frac{12}{5} i+\frac{13}{40}-j_{2}
\end{gathered}
$$

For all $x_{1}>0, x_{2}>0,\left|x_{1}^{-3 / 10} x_{2}{ }^{-6 / 5}\right|>1$. Thus, we can determine the 13-th order partial derivative value of $p\left(x_{1}, x_{2}\right)$ at $\left(\frac{4}{9}, \frac{1}{3}\right)$,

$$
\begin{gathered}
\frac{\partial^{13} p}{\partial x_{2}{ }^{4} \partial x_{1}{ }^{9}}\left(\frac{4}{9}, \frac{1}{3}\right) \\
=\sum_{i=0}^{\infty} \frac{\left(\begin{array}{c}
2 i \\
i
\end{array}\right)}{4^{i}(2 i+1)}\left(\frac{3}{5} i+\frac{24}{5}\right)_{9}\left(\frac{12}{5} i+\frac{13}{40}\right)_{4} \cdot\left(\frac{4}{9}\right)^{\frac{3}{5} i-\frac{21}{5}} \cdot\left(\frac{1}{3}\right)^{\frac{12}{5} i-\frac{147}{40}}
\end{gathered}
$$

Verifying the correctness of (21) using Maple.

$>\mathrm{p}:=(\mathrm{x} 1, \mathrm{x} 2)->\mathrm{x} 1^{\wedge}(9 / 2) * \mathrm{x} 2^{\wedge}(-7 / 8) * \operatorname{arccsc}\left(\mathrm{x} 1^{\wedge}(-3 / 10) * \mathrm{x} 2^{\wedge}(-6 / 5)\right)$; 


$$
p:=(x 1, x 2) \rightarrow \frac{x 1^{9 / 2} \operatorname{arccsc}\left(\frac{1}{x 1^{3 / 10} x 2^{6 / 5}}\right)}{x 2^{7 / 8}}
$$

$>\operatorname{evalf}(\mathrm{D}[1 \$ 9,2 \$ 4](\mathrm{p})(4 / 9,1 / 3), 14)$;

$$
-1.9977875714190 \cdot 10^{5}
$$

$>\operatorname{evalf}\left(\operatorname{sum}\left(1 /\left(4 \wedge^{\wedge} *(2 * \mathrm{i}+1)\right) *(2 * \mathrm{i}) ! /(\mathrm{i} ! * \mathrm{i} !) * \operatorname{product}(3 * \mathrm{i} / 5+24 / 5-\mathrm{j}, \mathrm{j}=0 . .8) * \operatorname{product}(12 * \mathrm{i} / 5+13 / 40-\mathrm{p}, \mathrm{p}=0 . .3)^{*}(4 / 9)^{\wedge}(3 * \mathrm{i} / 5-21 / 5)\right.\right.$ $*(1 / 3)^{\wedge}(12 * \mathrm{i} / 5-147 / 40), \mathrm{i}=0$.. infinity $\left.), 14\right)$;

$$
-1.9977875714184 \cdot 10^{5}
$$

3.4. Example 4 Let the domain of the three-variables function

$$
q\left(x_{1}, x_{2}, x_{3}\right)=x_{1}{ }^{-4 / 3} x_{2}{ }^{5 / 8} x_{3}{ }^{9 / 7} \sec ^{-1}\left(x_{1}{ }^{1 / 6} x_{2}{ }^{-3 / 11} x_{3}{ }^{-7 / 2}\right)
$$

be $\left\{\left(x_{1}, x_{2}, x_{3}\right) \in R^{3}\left|x_{1}>0, x_{2}>0, x_{3}>0,\right| x_{1}{ }^{1 / 6} x_{2}{ }^{-3 / 11} x_{3}{ }^{-7 / 2} \mid>1\right\}$. Using Theorem 4, we obtain any $j_{1}+j_{2}+j_{3}$-th order partial derivative of $q\left(x_{1}, x_{2}, x_{3}\right)$,

$$
\begin{gathered}
\frac{\partial^{j_{1}+j_{2}+j_{3}} q}{\partial x_{3}{ }^{{ }^{3}} \partial x_{2}{ }^{j_{2}} \partial x_{1}{ }^{j_{1}}}\left(x_{1}, x_{2}, x_{3}\right) \\
=\frac{\pi}{2} \cdot\left(\frac{-4}{3}\right)_{j_{1}}\left(\frac{5}{8}\right)_{j_{2}}\left(\frac{9}{7}\right)_{j_{3}} x_{1} \frac{-4}{3}-j_{1} \cdot x_{2} \frac{5}{8}-j_{2} \cdot x_{3} \frac{9}{7}-j_{3} \\
-\sum_{i=0}^{\infty} \frac{\left(\begin{array}{c}
2 i \\
i
\end{array}\right)}{4^{i}(2 i+1)}\left(-\frac{1}{3} i-\frac{3}{2}\right)_{j_{1}}\left(\frac{6}{11} i+\frac{79}{88}\right)_{j_{2}}\left(7 i+\frac{67}{14}\right)_{j_{3}} \cdot x_{1}^{-\frac{1}{3} i-\frac{3}{2}-j_{1}} \cdot x_{2} \frac{6}{11} i+\frac{79}{88}-j_{2} \cdot x_{3}{ }^{7 i+\frac{67}{14}-j_{3}}
\end{gathered}
$$

For all $x_{1}>0, x_{2}>0, x_{3}>0,\left|x_{1}{ }^{1 / 6} x_{2}{ }^{-3 / 11} x_{3}{ }^{-7 / 2}\right|>1$. Therefore, we can evaluate the 16 -th order partial derivative value of $q\left(x_{1}, x_{2}, x_{3}\right)$ at $\left(\frac{9}{8}, \frac{3}{4}, \frac{2}{5}\right)$,

$$
\begin{gathered}
\frac{\partial^{16} q}{\partial x_{3}{ }^{5} \partial x_{2}{ }^{7} \partial x_{1}{ }^{4}}\left(\frac{9}{8}, \frac{3}{4}, \frac{2}{5}\right) \\
=\frac{\pi}{2} \cdot\left(\frac{-4}{3}\right)_{4}\left(\frac{5}{8}\right)_{7}\left(\frac{9}{7}\right)_{5} \cdot\left(\frac{9}{8}\right)^{-\frac{16}{3}} \cdot\left(\frac{3}{4}\right)^{-\frac{51}{8}} \cdot\left(\frac{2}{5}\right)^{-\frac{26}{7}} \\
-\sum_{i=0}^{\infty} \frac{\left(\begin{array}{c}
2 i \\
i
\end{array}\right)}{4^{i}(2 i+1)}\left(-\frac{1}{3} i-\frac{3}{2}\right)_{4}\left(\frac{6}{11} i+\frac{79}{88}\right)_{7}\left(7 i+\frac{67}{14}\right)_{5} \cdot\left(\frac{9}{8}\right)^{-\frac{1}{3} i-\frac{11}{2}} \cdot\left(\frac{3}{4}\right)^{\frac{6}{11} i-\frac{537}{88}} \cdot\left(\frac{2}{5}\right)^{7 i-\frac{3}{14}}
\end{gathered}
$$

Also, we use Maple to verify the correctness of (24).

$>\mathrm{q}:=(\mathrm{x} 1, \mathrm{x} 2, \mathrm{x} 3)->\mathrm{x} 1^{\wedge}(-4 / 3)^{*} \mathrm{x} 2^{\wedge}(5 / 8)^{*} \mathrm{x} 3^{\wedge}(9 / 7) * \operatorname{arcsec}\left(\mathrm{x} 1^{\wedge}(1 / 6)^{*} \mathrm{x} 2^{\wedge}(-3 / 11)^{*} \mathrm{x} 3^{\wedge}(-7 / 2)\right)$;

$$
q:=(x 1, x 2, x 3) \rightarrow \frac{x 2^{5 / 8} x 3^{9 / 7} \operatorname{arcsec}\left(\frac{x 1^{1 / 6}}{x 2^{3 / 11} x 3^{7 / 2}}\right)}{x 1^{4 / 3}}
$$

$>\operatorname{evalf}(\mathrm{D}[1 \$ 4,2 \$ 7,3 \$ 5](\mathrm{q})(9 / 8,3 / 4,2 / 5), 14)$ 


\section{$-5.8286639095736 \cdot 10^{5}$}

$>$ evalf(Pi/2*product(-4/3-r, $\mathrm{r}=0 . .3) * \operatorname{product}(5 / 8-\mathrm{s}, \mathrm{s}=0 . .6)^{*} \operatorname{product}(9 / 7-\mathrm{t}, \mathrm{t}=0 . .4)^{*}(9 / 8)^{\wedge}(-16 / 3)^{*}(3 / 4)^{\wedge}(-51 / 8)^{*}(2 / 5)^{\wedge}(-26 / 7)-$ $\operatorname{sum}\left(1 /\left(4 \wedge^{\wedge} *(2 * \mathrm{i}+1)\right) *(2 * \mathrm{i}) ! /(\mathrm{i} ! * \mathrm{i} !) * \operatorname{product}(-\mathrm{i} / 3-3 / 2-\mathrm{j}, \mathrm{j}=0 . .3) * \operatorname{product}(6 * \mathrm{i} / 11+79 / 88-\mathrm{p}, \mathrm{p}=0 . .6) * \operatorname{product}(7 * \mathrm{i}+67 / 14-\mathrm{q}, \mathrm{q}=0 . .4)\right.$ $*(9 / 8)^{\wedge}(-\mathrm{i} / 3-11 / 2) *(3 / 4)^{\wedge}(6 * \mathrm{i} / 11-537 / 88) *(2 / 5)^{\wedge}(7 * \mathrm{i}-3 / 14), \mathrm{i}=0 .$. infinity $\left.), 14\right)$;

$$
-5.8286639095737 \cdot 10^{5}
$$

\section{Conclusion}

As mentioned, the differentiation term by term theorem plays a significant role in the theoretical inferences of this study. In fact, the application of this theorem is extensive, and can be used to easily solve many difficult problems; we endeavor to conduct further studies on related applications. On the other hand, Maple also plays a vital assistive role in problem-solving. In the future, we will extend the research topic to other calculus and engineering mathematics problems and solve these problems by using Maple. These results will be used as teaching materials for Maple on education and research to enhance the connotations of calculus and engineering mathematics.

\section{REFERENCES}

[1] C. T. J. Dodson and E. A. Gonzalez, Experiments in Mathematics Using Maple, New York: Springer-Verlag, 1995.

[2] D. Richards, Advanced Mathematical Methods with Maple, New York: Cambridge University Press, 2002.

[3] F. Garvan, The Maple Book, London: Chapman \& Hall/CRC, 2001.

[4] J. S. Robertson, Engineering Mathematics with Maple, New York: McGraw-Hill, 1996.

[5] R. J. Stroeker and J. F. Kaashoek, Discovering Mathematics with Maple : An Interactive Exploration for Mathematicians, Engineers and Econometricians, Basel: Birkhauser Verlag, 1999.

[6] C. Tocci and S. G. Adams, Applied Maple for Engineers and Scientists, Boston: Artech House, 1996.

[7] M. L. Abell and J. P. Braselton, Maple by Example, 3rd ed., New York: Elsevier Academic Press, 2005.

[8] D. N. Richard, An efficient method for the numerical evaluation of partial derivatives of arbitrary order, ACM Transactions on Mathematical Software, Vol. 18, No. 2, pp. 159-173, 1992.

[9] C. H., Bischof, G. Corliss, and A. Griewank, Structured second and higher-order derivatives through univariate
Taylor series, Optimization Methods and Software, Vol. 2, pp. 211-232, 1993.

[10] A. Griewank and A. Walther, Evaluating Derivatives: Principles and Techniques of Algorithmic Differentiation, 2nd ed., SIAM, Philadelphia, 2008.

[11] T-W, Ma, Higher chain formula proved by combinatorics, The Electronic Journal of Combinatorics , Vol. 16, \#N21, 2009.

[12] L. E. Fraenkel, Formulae for high derivatives of composite functions, Mathematical Proceedings of the Cambridge Philosophical Society, Vol. 83, pp. 159-165, 1978.

[13] C.-H. Yu, Using Maple to study the partial differential problems, Applied Mechanics and Materials, in press.

[14] C. -H. Yu, Partial derivatives of some types of two-variables functions, Pure and Applied Mathematics Journal, Vol. 2, No. 2, pp. 56-61, 2013.

[15] C. -H. Yu, Evaluating partial derivatives of two-variables functions by using Maple, Proceedings of the 6th IEEE/International Conference on Advanced Infocomm Technology, Taiwan, No. 00295, 2013.

[16] C.-H. Yu, Application of Maple on the partial differential problem of four types of two-variables functions, Proceedings of the International Conference on Advanced Information Technologies, Taiwan, No.87, 2013.

[17] C.-H. Yu, Application of Maple: taking the partial differential problem of some types of two-variables functions as an example, Proceedings of the International Conference on e-Learning, Taiwan, pp. 337-345, 2013.

[18] C. -H. Yu, Using Maple to evaluate the partial derivatives of two-variables functions, International Journal of Computer Science and Mobile Computing, Vol. 2, Issue. 6, pp. 225-232, 2013.

[19] C.-H., Yu, Application of Maple: taking the partial differential problem of two-variables functions as an example, Proceedings of 2013 Business Innovation and Development Symposium, Taiwan, B20130113001, 2013.

[20] Inverse trigonometric functions, online available from http://en.wikipedia.org/wiki/inverse_trigonometric_functions

[21] T. M. Apostol, Mathematical Analysis, 2nd ed., Boston: Addison-Wesley, p230, 1975. 